\title{
Bistable attractors in a model of convection-driven spherical dynamos
}

\author{
Radostin D Simitev ${ }^{1,3}$ and Friedrich H Busse ${ }^{2,3}$ \\ ${ }^{1}$ School of Mathematics and Statistics, University of Glasgow - Glasgow G12 8QW, UK, EU \\ ${ }^{2}$ Institute of Physics, University of Bayreuth - Bayreuth D-95440, Germany, EU \\ ${ }^{3}$ NORDITA, AlbaNova University Center - Stockholm SE-10691, Sweden, EU
}

D E-mail: Radostin.Simitev@glasgow.ac.uk

\section{Abstract}

The range of existence and the properties of two essentially different chaotic attractors found in a model of nonlinear convection-driven dynamos in rotating spherical shells are investigated. A hysteretic transition between these attractors is established as a function of the rotation parameter $\tau$. The width of the basins of attraction is also estimated.

Keywords: self-consistent MHD dynamos, thermal convection, oscillatory dynamos

\section{Introduction}

Chaotic systems by virtue of their apparently "random" fluctuations, are expected to frequent, with little bias, all points in their phase space over wide parameter ranges. This view implies that there should not be any abrupt transitions between distinguishable chaotic states. Indeed, non-linear transitions between attractors are rarely found in turbulent fluids. Contrary to this expectation, a number of examples of discontinuous transitions have been observed recently in chaotic and turbulent fluid systems [1-4]. Bifurcations between distinguishable 'chaotic states appear to be a little less unusual in magnetohydrodynamic flows because of the additional degrees of freedom offered by the magnetic field. For instance, various types of dipolar, quadrupolar, hemispherical dynamos, and bifurcations between them are routinely reported in numerical simulations, e.g. $[5,6]$. In turn, dipolar dynamos are typically found to belong to two distinct regimes - a regime with strong dipolar field, and another regime with weaker dipolar component and significant multipole contributions e.g. [6,7].

Far more remarkable is a recent finding that two essentially different chaotic dipolar dynamo solutions may exist at identical values of the basic parameters of a generic model of convection-driven dynamos in rotating spherical shells [8]. Such bistability offers the possibility of a hysteretic transition. While hysteresis was established in [8] as a function of three of the parameters in the problem - the Rayleigh, the ordinary, and the magnetic Prandtl numbers, to be defined below, the dependence on the last remaining basic parameter, the Coriolis number $\tau$, was not studied there. This, however, leaves an important gap since the variation in $\tau$ in this minimal self-consistent model of spherical convective dynamos describes the different rotation rates characteristic for various cosmic objects. In addition, given that current geodynamo simulations are unable to achieve geophysically realistic values of $\tau$, extrapolation of the $\tau$ dependence is heavily used to compare models and observations. As a partial remedy, bistability has been demonstrated for two specific values of $\tau$ in [9]. For these reasons, here we wish to investigate the full extent of the coexistence and hysteresis in dependence of the rotation parameter $\tau$. A number of other important questions left open in [8,9], including the width of the basins of attraction of the distinguishable chaotic states and the possibility of spontaneous transition will also be discussed in the present paper, along with results on essential properties of dynamo action such as kinetic, magnetic and cross-helicity generation.

\section{Formulation and methods}

\subsection{Model}

We employ a minimal model of nonlinear convection-driven dynamo process in rotating spherical shells with the aim to retain general applicability of the results to a variety of cosmic objects and to understand fundamental physical mechanisms. The model is identical to the one described in [8] but for completeness we provide a concise formulation below. We consider a spherical fluid shell of thickness $d$ rotating with a constant angular velocity $\Omega$. The existence of a static state is assumed with a temperature distribution $T_{S}=T_{0}-\beta d^{2} r^{2} / 2$ and a gravity field in the form $g=-d \gamma \boldsymbol{r}$, where $r d$ is the length of the position vector with respect to the center of the sphere. This form of temperature profile alludes to the possibility that at least a fraction of the energy available to planetary dynamos is due to radiogenic 
heat release. In addition to $d$, we use the time $d^{2} / v$, the temperature $v^{2} / \gamma \alpha d^{4}$ and the magnetic flux density $v(\mu \varrho)^{1 / 2} / d$ as scales for the dimensionless description of the problem where $v$ denotes the kinematic viscosity of the fluid, $\kappa$ its thermal diffusivity, $\varrho$ its density and $\mu$ its magnetic permeability. In common with most other simulations of Earth and planetary dynamos $[10,11]$, we assume the Boussinesq approximation implying a constant density $\varrho$ except in the gravity term where its temperature dependence is taken into account with $\alpha \equiv-(\mathrm{d} \varrho / \mathrm{d} T) / \varrho=$ const. The equations of motion for the velocity vector $\boldsymbol{u}$, the heat equation for the deviation $\Theta$ from the static temperature distribution, and the equation of induction for the magnetic flux density $\boldsymbol{B}$ are then given by

$$
\begin{gathered}
\nabla \cdot \boldsymbol{u}=0, \quad \nabla \cdot \boldsymbol{B}=0, \\
\left(\partial_{t}+\boldsymbol{u} \cdot \nabla\right) \boldsymbol{u}+\tau \boldsymbol{k} \times \boldsymbol{u}=-\nabla \pi+\Theta \boldsymbol{r}+\nabla^{2} \boldsymbol{u}+\boldsymbol{B} \cdot \nabla \boldsymbol{B}, \\
P\left(\partial_{t} \Theta+\boldsymbol{u} \cdot \nabla \Theta\right)=R \boldsymbol{r} \cdot \boldsymbol{u}+\nabla^{2} \Theta, \\
\nabla^{2} \boldsymbol{B}=P_{m}\left(\partial_{t} \boldsymbol{B}+\boldsymbol{u} \cdot \nabla \boldsymbol{B}-\boldsymbol{B} \cdot \nabla \boldsymbol{u}\right),
\end{gathered}
$$

where all gradient terms in the equation of motion have been combined into $\nabla \pi$. The dimensionless parameters in our formulation are the Rayleigh number $R$, the Coriolis number $\tau$, the Prandtl number $P$ and the magnetic Prandtl number $P_{m}$,

$$
R=\frac{\alpha \gamma \beta d^{6}}{v \kappa}, \quad \tau=\frac{2 \Omega d^{2}}{v}, \quad P=\frac{v}{\kappa}, \quad P_{m}=\frac{v}{\lambda},
$$

where $\lambda$ is the magnetic diffusivity. Being solenoidal vector fields, $\boldsymbol{u}$ and $\boldsymbol{B}$ can be represented uniquely in terms of poloidal and toroidal components,

$$
\begin{aligned}
& \boldsymbol{u}=\nabla \times(\nabla v \times \boldsymbol{r})+\nabla w \times \boldsymbol{r}, \\
& \boldsymbol{B}=\nabla \times(\nabla h \times \boldsymbol{r})+\nabla g \times \boldsymbol{r} .
\end{aligned}
$$

We assume fixed temperatures at $r=r_{i} \equiv 2 / 3$ and $r=r_{o} \equiv 5 / 3$ and stress-free rather than no-slip boundary conditions in order to approach, at least to some extent, the extremely low values of viscosity believed to be appropriate to planetary cores [12],

$$
v=\partial_{r r}^{2} v=\partial_{r}(w / r)=\Theta=0 .
$$

Two conditions on the poloidal scalar $v$ are required at each boundary because the corresponding poloidal equation is obtained by taking $\boldsymbol{r} \cdot \nabla \times \nabla \times$ of (1b) and thus it is of higher order as discussed below. For the magnetic field we assume electrically insulating boundaries at $r=r_{i}$ and $r=r_{o}$ such that the poloidal function $h$ matches the function $h^{(e)}$ which describes the potential fields outside the fluid shell,

$$
g=h-h^{(e)}=\partial_{r}\left(h-h^{(e)}\right)=0 \text { at } r=r_{i}, r_{o} .
$$

The radius ratio $r_{i} / r_{o}=0.4$ is slightly larger than that appropriate for the Earth's liquid core. This is a standard formulation of the spherical convection-driven dynamo problem $[10,11,13]$ for which an extensive collection of results already exists $[5,6,14,15]$. The results reported below are not strongly model dependent. In particular, dynamos with stress-free and with no-slip velocity boundary conditions as well as with different modes of energy supply are known to have comparable energy densities and symmetry properties (see fig. 15 of [11]).

\subsection{Methods of solution}

Equations of motion for the scalar fields $v, w$, are obtained by taking $\boldsymbol{r} \cdot \nabla \times \nabla \times$ and $\boldsymbol{r} \cdot \nabla \times$ of equation (1b) and equations for $g$ and $h$ are obtained by taking $\boldsymbol{r} \cdot \nabla \times$ and $\boldsymbol{r}$. of equation (1d). These equations are solved numerically by a pseudospectral method as described in [16] based on expansions of all dependent variables in spherical harmonics for the angular dependences and in Chebychev polynomials for the radial dependence. A minimum of 41 collocation points in the radial direction and spherical harmonics up to the order 96 have been used in all cases reported here which provides adequate resolution The dynamo solutions are characterized by their magnetic energy densities,

$$
\begin{array}{ll}
\bar{M}_{p}=\frac{1}{2}\left\langle|\nabla \times(\nabla \bar{h} \times \boldsymbol{r})|^{2}\right\rangle, & \bar{M}_{t}=\frac{1}{2}\left\langle|\nabla \bar{g} \times \boldsymbol{r}|^{2}\right\rangle, \\
\widetilde{M}_{p}=\frac{1}{2}\left\langle|\nabla \times(\nabla \widetilde{h} \times \boldsymbol{r})|^{2}\right\rangle, & \widetilde{M}_{t}=\frac{1}{2}\left\langle|\nabla \widetilde{g} \times \boldsymbol{r}|^{2}\right\rangle,
\end{array}
$$

where $\langle\cdot\rangle$ indicates the average over the fluid shell and $\bar{h}$ refers to the axisymmetric component of $h$, while $\widetilde{h}$ is defined by $\widetilde{h}=$ $h-\bar{h}$. The corresponding kinetic energy densities $\bar{E}_{p}, \bar{E}_{t}, \widetilde{E}_{p}$ and $\widetilde{E}_{t}$ are defined analogously with $v$ and $w$ replacing $h$ and $g$. The total magnetic energy density is $M=\bar{M}_{p}+\bar{M}_{t}+\widetilde{M}_{p}+\widetilde{M}_{t}$, and similarly for the total kinetic energy density $E$. In addition, the magnetic energy densities can be divided into those of fields that are antisymmetric (axial dipole symmetry) and those that are symmetric (axial quadrupole symmetry) with respect to the equatorial plane. The former (latter) are described by spherical harmonics $Y_{l}^{m}$ with odd (even) $l+m$. Other quantities of interest are the helicity density of a vector field $\boldsymbol{a}$,

$$
H_{a}=\boldsymbol{a} \cdot(\nabla \times a)
$$

(known as "kinetic" helicity density when $\boldsymbol{a}=\boldsymbol{u}$, and "magnetic" helicity density when $\boldsymbol{a}=\boldsymbol{B}$, respectively [17]), and the cross-helicity density

$$
H_{\times}=\boldsymbol{u} \cdot \boldsymbol{B},
$$

all of which play important roles in the production of the magnetic field by the chaotic convective flow.

\section{Bistability}

Typical examples of solutions to the dynamo problem outlined above are shown in Figure 1. The figure presents the main magnetic and kinetic energy density components of two distinct dynamo cases as functions of time, and illustrates well the chaotic nature of the solutions. Apart from the obvious quantitative difference, an essential qualitative change in the balance of magnetic energy components can be observed. The axisymmetric poloidal component $\bar{M}_{p}$ is dominant in the case shown in Figure 1 $(a, b)$ while it has a relatively small contribution in the case of Figure 1(d,e). This observation is in agreement with the claim made in $[5-8,18,19]$ that, in general, two regimes of dipolar dynamos can be distinguished, namely those with $\widetilde{M}_{p}<\bar{M}_{p}$ (denoted by MD, "Mean Dipole" in [8] and below) 
MD

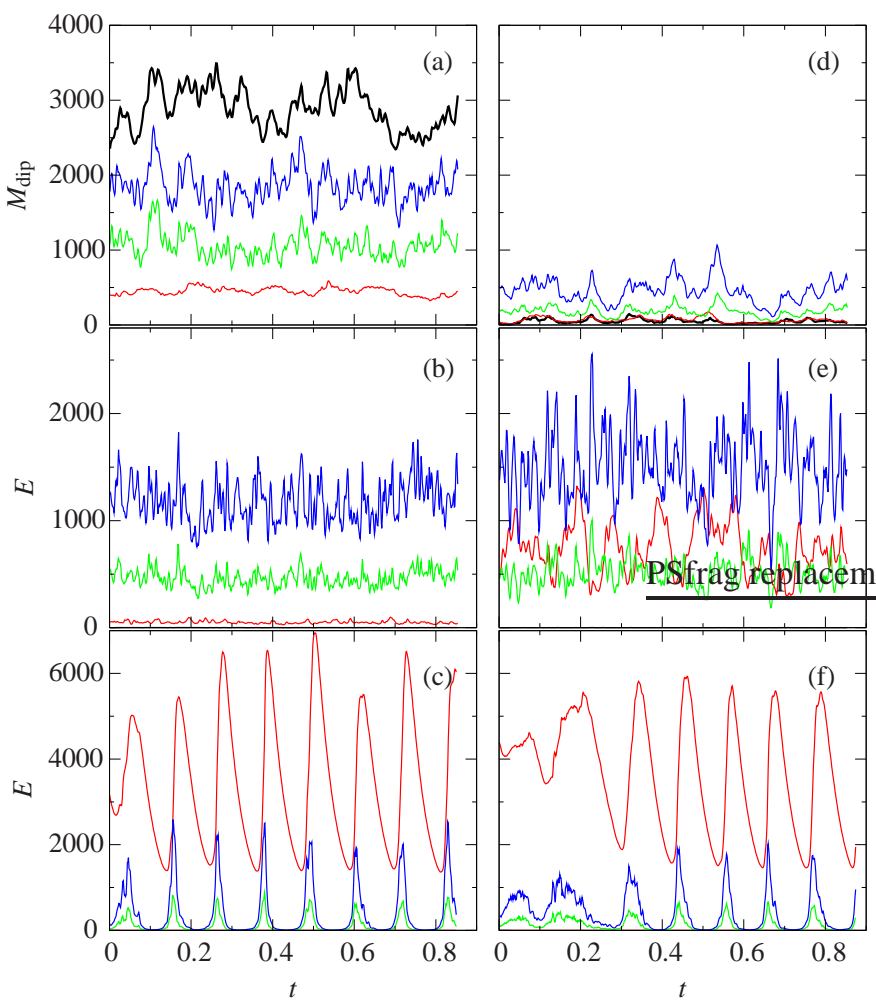

Figure 1: (color online) Coexisting distinct chaotic dynamo attractors at identical parameter values - a MD (left column $(\mathrm{a}, \mathrm{b})$ ) and a FD dynamo (right column (d,e)) both at $R=1.5 \times 10^{6}, \tau=2 \times 10^{4}, P=0.75$ and $P_{m}=1.5$. Panels $(\mathrm{a}, \mathrm{d})$ show time series of magnetic dipolar energy densities. The rest of the panels show kinetic energy densities in the presence of magnetic field (b,e) and after the magnetic field is removed (c,f). The component $\bar{X}_{p}$ is shown by solid black line, while $\bar{X}_{t}, \widetilde{X}_{p}$, and $\widetilde{X}_{t}$ are shown by red, green and blue lines, respectively. $X$ stands for either $M$ or $E$.

and those with $\widetilde{M}_{p}>\bar{M}_{p}$ (denoted by FD, "Fluctuating Dipole" in [8] and below). The dynamos in Figure $1(\mathrm{a}, \mathrm{b})$ and $1(\mathrm{~d}, \mathrm{e})$ are examples of these two types. A convenient measure of the type of dynamo is therefore provided by the ratio $\widetilde{M}_{p} / \bar{M}_{p}$, which we use extensively below.

Far more remarkable is the fact that the two distinct solutions shown in Figure 1 are obtained at identical parameter values and coexist in this case. In fact, this is far from being an isolated example. Indeed, varying the value of $\tau$ we find an extended region of coexisting MD and FD dynamos as illustrated in Figure 2(a) where the ratio $\widetilde{M}_{p} / \bar{M}_{p}$ is plotted as a function of $\tau$ in the case $P=0.75, P_{m}=1.5$ and $R \approx 4 \times R_{c}$, where $R_{c}$ is the critical Rayleigh number for the onset of convection. The transition between the MD and FD dynamos is discontinuous and it is achieved via a hysteresis loop in the following sense. When an MD dynamo is used as initial data and the Coriolis number $\tau$ is gradually decreased, solutions remain in regime $\mathbf{M D}$ until the critical value $\tau_{\mathrm{FD}} \approx 12500$ is reached at which point an abrupt jump transition to the FD regime occurs. Similarly, when a FD dynamo is used as an initial condition and $\tau$ is gradually increased the reverse transition occurs at the critical value $\tau_{\mathrm{MD}} \approx 39000$ as seen in Figure 2(a). The solutions plotted in Figure 2 have been typically continued at least up to 4 magnetic diffusion times. No evidence for a transient nature in

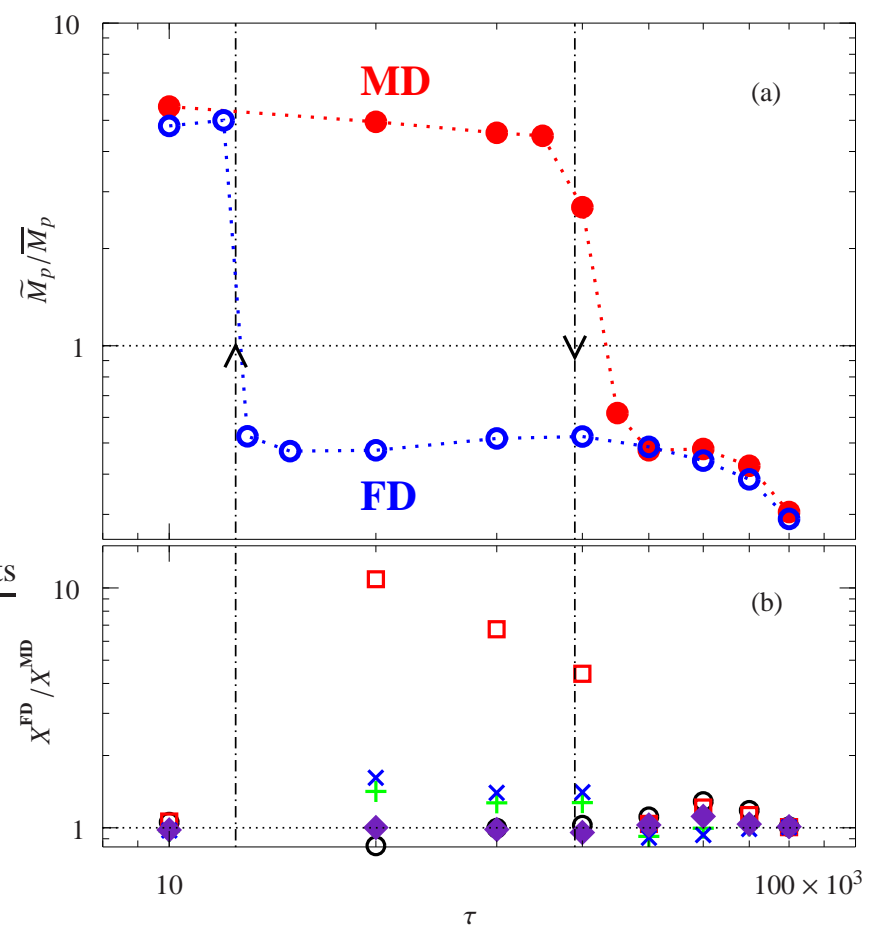

Figure 2: (color online) (a) Coexistence and hysteresis phenomena shown by the time-averaged ratio $\widetilde{M}_{p} / \bar{M}_{p}$ as a function of $\tau$. FD and MD dynamos are indicated by full red and empty blue circles, respectively. Expected locations of the transitions from FD to MD dynamos, and vice versa are indicated by dash-dotted lines with arrows pointing down and up, respectively. (b) Comparison of convective properties of FD and MD dynamos measured by the rations $N u_{i}^{\mathrm{FD}} / N u_{i}^{\mathrm{MD}}$ (violet diamonds), $\bar{E}_{p}^{\mathrm{FD}} / \bar{E}_{p}^{\mathrm{MD}}$ (black circles), $\bar{E}_{t}^{\mathrm{FD}} / \bar{E}_{t}^{\mathrm{MD}}$ (red squares), $\widetilde{E}_{p}^{\mathrm{FD}} / \widetilde{E}_{p}^{\mathrm{MD}}$ (green plus signs), $\widetilde{E}_{t}^{\mathrm{FD}} / \widetilde{E}_{t}^{\mathrm{MD}}$ (blue crosses), all as functions of $\tau$. Parameter values are $P=0.75, P_{m}=1.5$ and $R=\left(5-3 \cdot 10^{-5} \tau\right) R_{c}$, i.e. $R \cdot 10^{-5}=7.6,17,26,35,43,51,58,62$ at $\tau=n \times 10^{4}, n=1, \ldots 8$.

any case has been found. In fact, in cases outside of the region of double attractors it takes typically less than 0.15 magnetic diffusion times to switch from the initial conditions used to the appropriate unique attractor.

The results shown in Figure 2(a) are an important complement to the findings of $[8,9]$ where coexistence and hysteresis was established as a function of the remaining non-dimensional parameters $P, P_{m}$ and $R$ for fixed values of $\tau=3 \times 10^{4}, 4 \times 10^{4}$ (see Figures 5 and 10 of [8], and [9], respectively).

\section{Comparison of bistable attractors: Magnetic features}

All solutions included in Figure 2 have a predominantly dipolar character with the ratio $\bar{M}_{p}^{\text {dip }} / \bar{M}_{p}$, quantifying dipolarity the field visible outside of the spherical shell, in the ranges $[0.93,1]$ for MD dynamos and [0.42,0.70] for FD dynamos, respectively. Although, the FD dynamos feature an increased contribution of higher multipoles they are of significant geophysical relevance $[6,18,19]$. In this section we wish to discuss some of the magnetic properties of MD to FD dynamos in more detail than it has been done previously in $[8,9]$. 


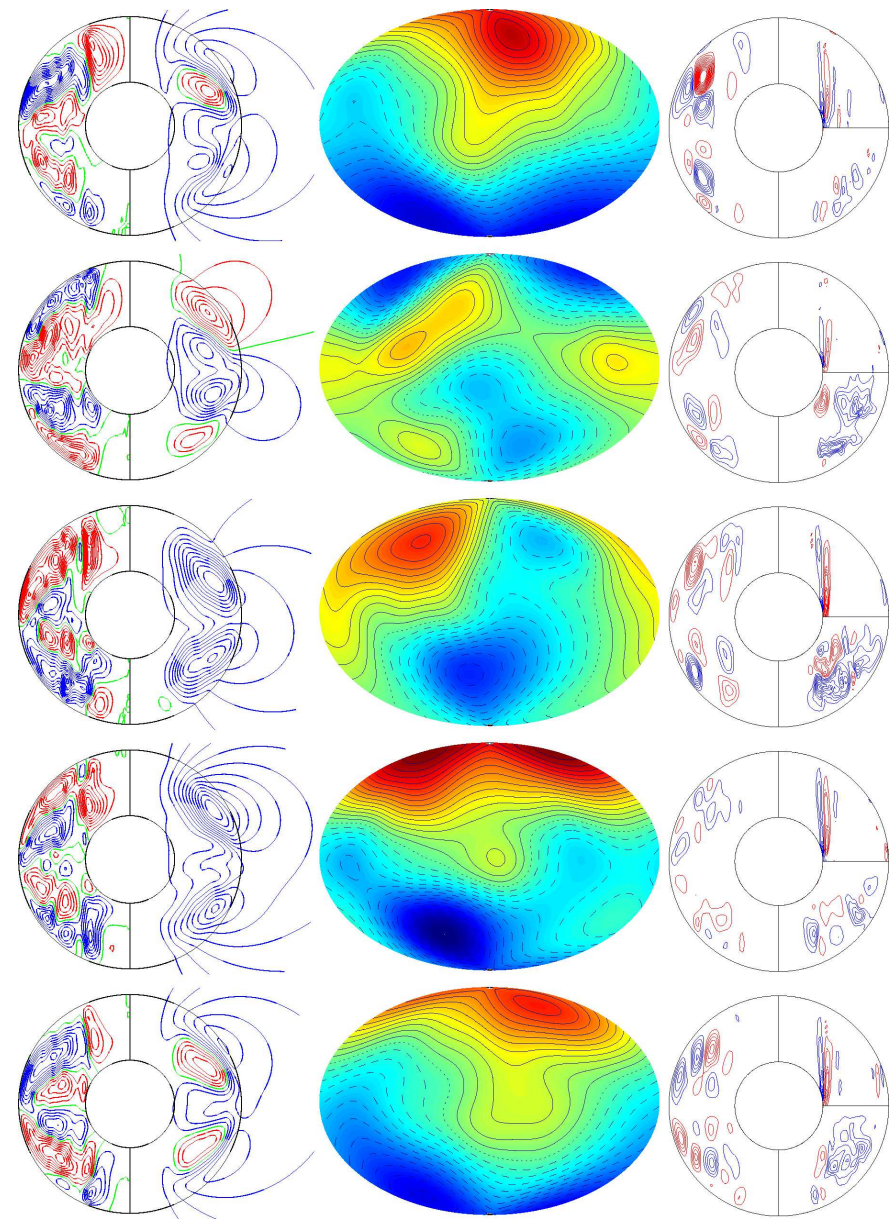

Figure 3: (color online) A period of dipolar oscillations of a FD dynamo. The plots in the leftmost column are meridional cuts showing lines of constant $\overline{B_{\varphi}}$ in the left half and of $r \sin \theta \partial_{\theta} \bar{h}$ in the right half. The plots in the middle column show lines of constant $B_{r}$ at $r=r_{o}+1.3$. The plots in the rightmost column are meridional cuts showing lines of constant densities of cross-helicity $H_{\times}$, kinetic helicity $H_{u}$ and magnetic helicity $H_{B}$ at $\varphi=0$, in the left half, right upper and right lower quarters, respectively. The rows correspond to equidistant moments separated by $\Delta t=0.0252$. The parameter values are the same as in Figure 1 .

\subsection{Time dependence}

Convection-driven dynamos exhibit chaotic time dependence (see e.g. Figure 1) except in simple cases close to the critical value of $R$ and for rather large values of $P_{m}$, such as the well-known dynamo benchmark case [20]. There are, however, some coherent temporal features that can be distinguished. In particular, dynamos in the FD regime are typically oscillatory in that nearly periodic changes in amplitude and field structures can be observed. As an illustration, Figure 3 shows a period of one such dipolar oscillation of the FD dynamo discussed in connection to Figure 1(d,e). At the beginning of the cycle magnetic flux with a "new" polarity is generated near the equator of the inner boundary, and subsequently propagates towards the poles replacing the flux of "old" polarity, as can be best seen in the plots of $\bar{B}_{\varphi}$ in this figure. The process repeats in a quasi-periodic fashion. Kinetic helicity density remains largely unaffected while the magnetic and the cross-helicity densities participate in the oscillation. On the other hand, dynamos in

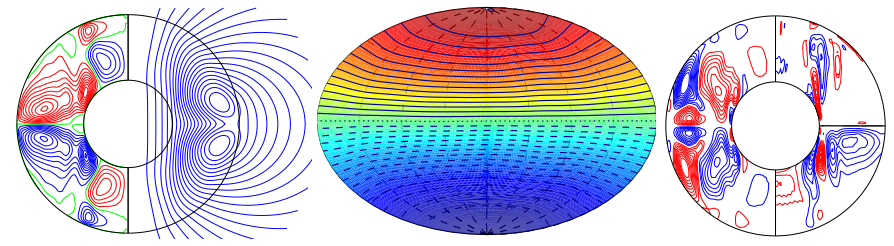

Figure 4: (color online) Time-averaged spatial structures of a MD dynamo. The same quantities are plotted as in Figure 3. The parameter values are the same as in Figure 1.

the MD regime show significantly less variation in time and feature spacial structures that remain nearly static and fluctuate little with respect to their time averages. This is due to the dominance of the mean components of the poloidal magnetic field characteristic for the MD regime.

\subsection{Spatial structures}

Typical time-averaged spatial structures in the MD regime are illustrated in Figure 4 by the example already discussed in connection to Figure 1(a,b). The dynamo exhibits a nearly perfect and relatively large-scale dipolar field best seen in the plots of the radial magnetic field and the meridional field lines $r \sin \theta \partial_{\theta} \bar{h}=$ const. In particular, two strong zonal magnetic flux tubes of $\overline{B_{\varphi}}$ are formed inside the tangent cylinder, near the poles, while two tubes of opposite polarity reside on both sides of the equator. The kinetic and magnetic helicity densities are generated in narrow plumes primarily at the boundary of the tangent cylinder, while the cross-helicity density forms strong azimuthal tubes of alternating polarity filling the region outside of the tangent cylinder. In contrast, the spatial structures of FD dynamos, which are exhibited in Figure 3, have relatively smaller scales and the evidence of higher multipole contributions are clearly visible. At the minimum of the dipolar oscillation the zonal magnetic flux tubes near the poles disappear, and the radial magnetic field shows an excursion towards the opposite polarity and an $m=1$ structure. The kinetic and magnetic helicity densities are again generated near the tangent cylinder but appear smaller-scale and more fragmented.

\section{Comparison of bistable attractors: Convective features}

\subsection{Non-magnetic convection}

The coexistence and hysteresis phenomena appear to be entirely magnetic in nature. This is nicely illustrated in Figure $1(\mathrm{c}, \mathrm{f})$ where the kinetic energy densities of two non-magnetic solutions are shown, which are started from initial conditions in the corresponding MD and FD dynamos in same figure. Once the magnetic field is discarded, the flow quickly equilibrates to the same purely convective state. At these values of the parameters, the convective state achieved is the well-known state of relaxation oscillations [15, 21]. This state of convection is remarkable in itself as it has a coherent nearly periodic behaviour in an otherwise chaotic regime. Evidence of its quasiperiodicity can be seen both in the time series of Figure 1(c,f) as well as in the period of relaxation oscillations shown in Figure 5 . 


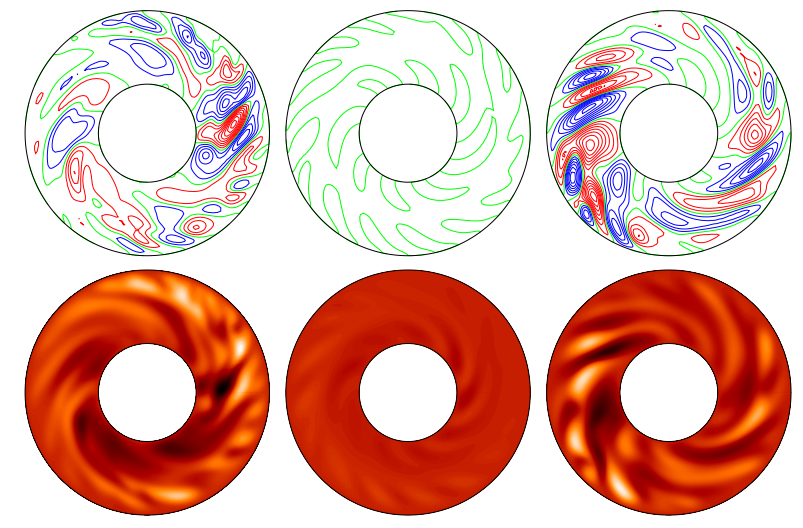

Figure 5: (color online) A period of relaxation oscillations seen in the equatorial plane of a non-magnetic solution. The top row shows streamlines $r \partial_{\varphi} v=$ const and the bottom row shows color maps of the temperature perturbation $\Theta$ both in the equatorial plane. The time step between plots is $\Delta t=0.0448$. The same case is shown in Figure 1(f).

Relaxation oscillations are one of a number of states achieved by turbulent convection with increase of the Rayleigh number $R$. In this state the differential rotation generated by the Reynolds stresses of the convection columns becomes so large that it is able to destroy all convective structures by shearing them off in the azimuthal direction. In the absence of convection the differential rotation must decay since there are no Reynolds stresses to sustain it. As the shearing action of the differential rotation becomes sufficiently weak convection columns grow in amplitude again. But as their Reynolds stresses regenerate the differential rotation, their amplitude quickly peaks and then decays as the shearing action interrupts the convection flows. It is surprising how nearly periodically this process repeats itself even though every convection episode differs from the next one in detail. The period of these relaxation oscillations is primarily governed by the viscous decay of the differential rotation. Since our study is based on the viscous decay time the value of about 0.1 was found for the period over a wide range of the parameters $R, \tau$, and $P$.

\subsection{Magnetic convection}

The properties of convection in the presence of magnetic field differ relatively little between dynamos in the FD and MD regime. The time dependence of the respective convective flows can be compared in panels (b) and (e) of Figure 1 where kinetic energy density components are shown. The time dependence is chaotic in both cases, and it may be noted that convection is not in the state of relaxation oscillations characteristic for the corresponding non-magnetic cases. While the average values of the fluctuating components $\widetilde{E}_{p}$ and $\widetilde{E}_{t}$ are nearly the same for the FD and MD case, the main difference is the significant increase in differential rotation measured by $\bar{E}_{t}$, which in the FD dynamo is about 20 times larger than in the MD dynamo. Spatial structures of convection for the two dynamos are shown in Figure 6. The length scales and the level of irregularity of the structures is very similar for the two regimes. The main difference appears in the profiles of the zonal flow, which is
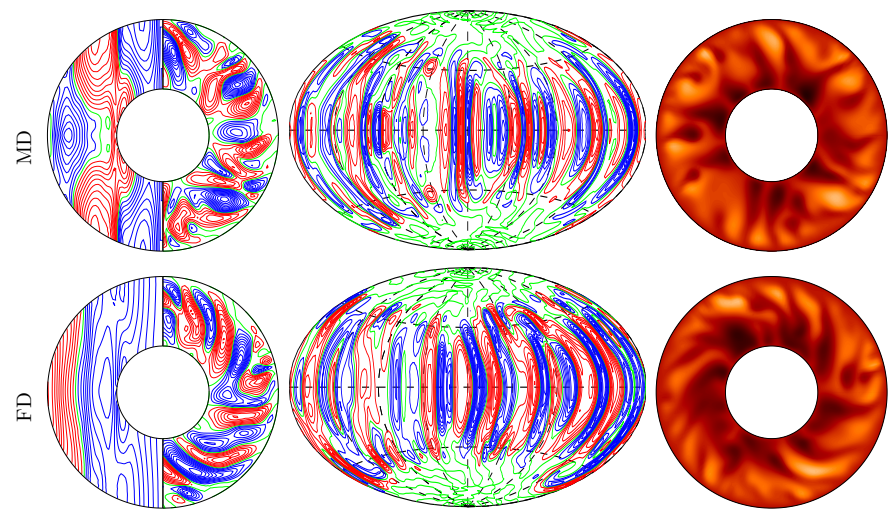

Figure 6: (color online) Spatial structures of convection of a MD and a FD dynamos. The leftmost plots show lines of constant $\bar{u}_{\varphi}$ in the meridional plane on the left, and streamlines $r \partial_{\varphi} v=$ const. in the equatorial plane on the right. The middle plots show lines of constant $u_{r}$ on the spherical surface $r=r_{i}+0.5$. The rightmost plots show color maps of the temperature perturbation $\Theta$ in the equatorial plane. The parameter values are the same as in Figure 1.

nearly constant in $z$, the direction parallel to the axis of rotation, in the FD dynamo and strongly dependent on $z$ in the MD case. In addition, while the differential rotation near the equator is in the prograde direction in the former case, it is in the retrograde direction in the latter one. It is well-known that the main effect of self-sustained magnetic field on convection is to strongly inhibit differential rotation, e.g. [5]. This effect is stronger in the case of MD dynamos which are characterized by stronger magnetic fields and explains the observed differences. Evidence that these differences are typical throughout the MD and FD regimes is presented in Figure 2(b) where ratios of various kinetic energy components as well as the Nusselt numbers are plotted for a number of coexisting attractors as a function of $\tau$, and it can be seen that their values do not differ significantly from unity except in the case of $\bar{E}_{t}^{F D} / \bar{E}_{t}^{M D}$.

\subsection{Mechanism of coexistence}

The observations just made are useful in elaborating the mechanism of the coexistence phenomenon. Coexistence is the result of two different ways in which the magnetic field damps the differential rotation to achieve the transport of the same amount of heat. In MD dynamos the differential rotation generated by Reynolds stresses of the convection columns is eliminated almost entirely by the strong mean magnetic field. Because of the strong magnetic field the amplitude of convection is also reduced in comparison with the maximum value that it reaches in the absence of a magnetic field. In the case of FD dynamos the differential rotation is still diminished, but its alignment with coaxial cylindrical surfaces is preserved. The amplitude of convection is now more strongly fluctuating, but is larger on average than in the case of the MD dynamos. In this way FD and MD dynamos manage to carry very nearly the same heat transport as is evident from Figure 2(b). This heat transport by far exceeds the time average of the heat transport found in the absence of a magnetic field. 


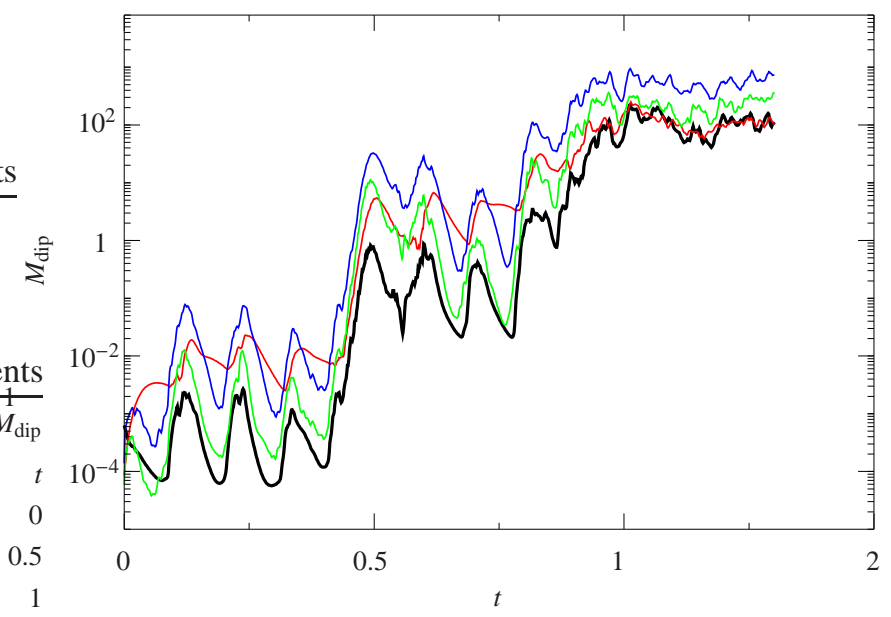

Figure 7: (color online) Equilibration of a dynamo started from a small random magnetic field seed. Time series of magnetic dipolar energy densities with the same color scheme as in Figure 1 are shown. The same parameter values as in Figure 1 are used.

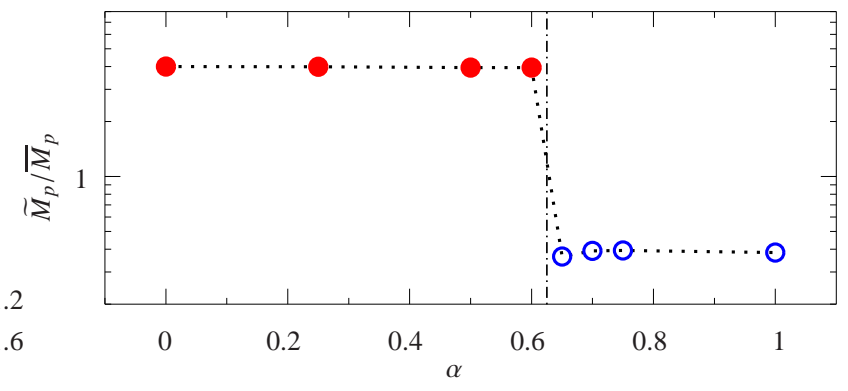

Figure 8: (color online) Width of the basins of attraction of MD and FD states shown by the ratio $\widetilde{M}_{p} / \bar{M}_{p}$ as a function of the continuation parameter $\alpha$ of formula (6), in the case $R=1.5 \times 10^{6}, \tau=2 \times 10^{4}, P=0.75$ and $P_{m}=1.5$. The cases with $\alpha=0$ and $\alpha=1$ are the same FD (red full circles) and the MD dynamos (blue empty circles) shown in Figure 1, respectively.

\section{Basins of attraction}

Usually a dynamo equilibrates to a unique state. In contrast, for a dynamo inside the region of coexistence, the initial conditions determine whether a FD or a MD state is approached, as discussed in connection with Figure 2(a). Here we attempt to estimate the set of initial conditions that leads to equilibration to a MD or a FD state.

\subsection{Random initial conditions}

Figure 7 shows a numerical experiment in which random magnetic perturbation of small amplitude is applied to fully developed convection in the regime of relaxation oscillations, and integration in time is continued for the velocity, temperature and magnetic fields. After a relatively long transient period of magnetic field growth, the dynamo approaches the FD regime. In addition, this simulation suggests that the existence of a third chaotic attractor along with the FD and the MD states is unlikely.

\subsection{Controlled initial conditions}

The vast majority of dynamo solutions published in the literature are started from previously equilibrated runs with similar parameter values in order to minimize transient times. In the region of coexistence a dynamo started from another FD (MD) dynamo approaches a FD (MD) state. In order to estimate the width of the basins of attraction of the two coexisting states, we report in Figure 8 a number of simulations started from initial conditions prepared in the form,

$$
x(r, \theta, \varphi)=\alpha x^{\mathrm{MD}}(r, \theta, \varphi)+(1-\alpha) x^{\mathrm{FD}}(r, \theta, \varphi),
$$

where $\alpha \in[0,1]$ is a continuation parameter, $x$ represents any of the dynamical variables $\boldsymbol{u}, \boldsymbol{B}$ and $\Theta$, and the superscripts indicate equilibrated FD and MD dynamo solutions. When $\alpha=0$ this is equivalent to initial conditions chosen in the FD regime, when $\alpha=1$ corresponds to initial conditions chosen in the MD regime, and variation of $\alpha \in(0,1)$ allows us to follow a continuous path between the two attractors. Figure 8 shows that for the test case $P=0.75, R=1.5 \times 10^{6}, \tau=2 \times 10^{4}$ and $P_{m}=1.5$ the transition between $\mathbf{F D}$ and $\mathbf{M D}$ regimes occurs at $\alpha=0.625 \pm 0.25$.

We wish to comment that while both Figures 7 and 8 show some bias towards the FD regime, this may be due to our inability to select a test case situated exactly in the middle of the coexistence region.

\section{Concluding remarks}

We have considered in this study a minimal self-consistent model of dynamo action generated by convection in rotating spherical fluid shells. While the relatively thick spherical shells, the relatively large values of the Coriolis parameter, and the relatively low values of the Rayleigh number employed here are more appropriate to the problem of geo- and planetary magnetism, the model is generic and may be used to understand solar and stellar magnetism. For instance, the periodic reversals characteristic for FD dynamos are reminiscent of the 11year Solar cycle. In particular, we have been concerned here with the possibility of coexistence of two nonlinear attractors in the fully-developed chaotic dynamo regime, and the hysteretic transition between them. These phenomena have been noted previously in [8,9] and discussed there in some detail. The following is a summary of the important points made here.

(a) We have established the coexistence of two nonlinear attractors, denoted by MD and FD dynamos in the above, over a significantly large interval of values of the Coriolis number $\tau \in(12500,39000)$. The transition between them takes the form of a hysteresis loop. These results fill a gap left in $[8,9]$ and demonstrate that coexistence occurs as a function of all basic parameters in the model.

(b) We have discussed in detail the contrasting properties characterizing dynamos in the MD and FD regimes, including differences in temporal behaviour and spatial structures of 
both magnetic field and convection. We include new results on quantities important in mean-field dynamo theories of magnetic field generation such as the kinetic, magnetic and cross-helicity density profiles in time-averages as well during oscillations and reversals.

(c) We have investigated the question of the width of the basins of attraction of the coexisting chaotic states.

The coexistence of two distinct turbulent attractors is also a phenomenon of general interest as it is relatively rare in fluid dynamics and magnetohydrodynamics. Finally, the range of values of the Coriolis number $\tau$ where we have found coexistence constitutes a rather large subinterval of the range currently accessible by numerical simulations. This requires extra care when numerical results are interpreted.

\section{Acknowledgements}

The research reported in the paper was performed in parts during the authors' participation in the 2011 program "Dynamo, Dynamical Systems and Topology" at NORDITA. The research of R. Simitev has also been supported by the UK Royal Society under Research Grant 2010 R2.

\section{References}

[1] F. Ravelet, L. Marié, A. Chiffaudel, F. Daviaud, Phys. Rev. Lett. 93 (2004) 164501.

[2] N. Mujica, D. Lathrop, J. Fluid Mech. 551 (2006) 49.

[3] M. Berhanu, et al., J. Fluid Mech. 641 (2009) 217-226.

[4] S. Weiss, R. Stevens, J. Zhong, H. Clercx, D. Lohse, G. Ahlers, Phys. Rev. Lett. 105 (2010) 224501.

[5] R. Simitev, F. H. Busse, J. Fluid Mech. 532 (2005) 365.

[6] F. H. Busse, R. Simitev, Geophys. Astrophys. Fluid Dyn. 100 (2006) 341.

[7] U. Christensen, J. Aubert, Geophys. J. Int. 166 (2006) 97.

[8] R. Simitev, F. Busse, EPL 85 (1) (2009) 19001+.

[9] F. H. Busse, R. Simitev, Vol. 28 of IUTAM Bookseries, Springer, 2010, pp. 181-194.

[10] E. Dormy, J.-P. Valet, V. Courtillot, Geochem. Geophys. Geosyst. 1 (2000) 2000GC000062.

[11] M. Kono, P. Roberts, Rev. Geophys. 40 (2002) 1013.

[12] W. Kuang, J. Bloxham, Nature 389 (1997) 371.

[13] F. H. Busse, Annu. Rev. Fluid Mech. 32 (2000) 383.

[14] E. Grote, F. H. Busse, A. Tilgner, Phys. Earth Planet. Inter. 117 (2000) 259.

[15] E. Grote, F. H. Busse, Fluid Dyn. Res. 28 (2001) 349.

[16] A. Tilgner, Int. J. Num. Meth. Fluids 30 (1999) 713.

[17] K. Moffatt, Magnetic Field Generation in Electrically Conducting Fluids, Cambridge, 1978.

[18] C. Kutzner, U. Christensen, Phys. Earth Planet. Inter. 131 (2002) 29.

[19] P. Olson, G. Glatzmaier, R. Coe, Earth Planet. Sci. Lett. 304 (2011) 168 -179 .

[20] U. Christensen, et al., Phys. Earth Planet Inter. 128 (1-4) (2001) 25-34.

[21] R. Simitev, F. Busse, New J. Phys. 5 (2003) 97. 